\title{
DIE SUBNIVALE HÖHENSTUFE UND IHRE UNTERGRENZE IN DEN BÜNDNER UND WALLISER ALPEN
}

\author{
Mit + Illustrationen \\ GERHARD FURRER
}

Einen wesentlichen Bestandteil der Hochgebirgslandschaft bildet neben der alpinen und der nivalen die subnivale Höhenstufe mit ihrem solifluidalen Formenkreis (Troll 1955). Nach A. Penck (1910) erstreckt sich dieser Bereich von der Schneegrenze bis ungefähr dorthin, wo die Schneebedeckung noch einen Monat dauert (Troll 1944, p. 557), und schließt so große Teile der Waldstufe mit ein. In seiner umfassenden Arbeit über «Strukturböden, Solifluktion und Frostklimate der Erde〉 (1944) faßt Troll den Begriff allerdings bedeutend enger und bezeichnet mit ssubnival〉 nur noch die kältere Unterprovinz des subnivalen Bereiches im Sinne von A. Penck, das heißt die jenseits der Baumgrenze nahe der nivalen Stufe liegenden Gebiete. Gleichzeitig stellt er die Frage, «ob man den subnivalen Bereich nicht überhaupt besser mit Hilfe des Bodenfrostes als mit der Schneedecke abgrenzt) (p. 557), und legt 1947 dar, daß es gelingt, eine deutliche klimatische Grenze der Strukturbodenbildung und der allgemeinen Solifluktion in der Natur festzulegen〉, «wobei wir eine polare Grenze und eine untere Höhengrenze in den Gebirgen unterscheiden können> (p. 163). Allerdings müssen zu diesem Zwecke die zonalen Erscheinungsformen sehr sorgfältig von allfälligen a- und extrazonalen Strukturbodenvorkommen geschieden werden (Troll 1944, p. 592).

Alle Klimate mit morphologisch wirksamer Gefrornis und Solifluktion lassen sich innerhalb des humiden und semiariden Bereiches zu einem besondern Klimabereich zusammenfassen (Troll 1948, p. 5 und Abb. 1). Diesen grenzt Troll in den Hochgebirgen nach unten durch die (klimatische Strukturbodengrenzes ab.

Der Kleinformenschatz zwischen der Baum- und der Schneegrenze ist in den Hochgebirgen Europas über große Räume hinweg untersucht worden. Deshalb zeichnet sich die Möglichkeit ab, mit Hilfe von Kleinformen, die durch Solifluktion und Bodenfrost gebildet werden, eine höhenzonale Gliederung der erwähnten Stufe vorzunehmen. Wie neuerdings gezeigt wurde (Furrer 1965), können in unsern Alpen eine Untergrenze der Strukturböden und eine solche der Formen der gebundenen Solifluktion festgelegt werden. Ich fasse die subnivale Höhenstufe als den Bereich zwischen der oberen Strukturbodengrenze, die im wesentlichen der Schneegrenze entsprechen sollte, und der Untergrenze der. Formen der gebundenen Solifluktion auf (Fig. 1).

In der Arktis wurde neuerdings wieder untersucht, wie diese beiden Formbildungsprozesse Frost und Solifluktion - im einzelnen wirken und ineinandergreifen (Büdel 1960 und 1961), und in den Schweizer Alpen laufen gegenwärtig Studien über die Materialabhängigkeit von Solifluktions- und Frostmusterböden an.

Untersuchungen über die Verbreitung des subnivalen Formenschatzes ergaben, daß zwischen Wald- und Schneeregion die einzelnen Formtypen bestimmte Höhenlagen bevorzugen. Daher läßt sich dieser Bereich in Stufen verschiedener subnivaler Kleinformen gliedern. Die Auswertung der Beobachtungen in den Bündner und den Walliser Alpen führte zum in Figur 1 wiedergegebenen Modell. Die Darstellung stützt sich ausschließlich auf zonale Vorkommen (Poser 1954, Furrer 1959 und 1965, Höllermann 1964). Extrazonale Phänomene, insbesondere Strukturbodenformen in Gletschervorfeldern, sind nicht berücksichtigt; denn im Vorfeld eines Gletschers bilden sich echte Strukturböden nur unter besonderen Bedingungen (Furrer 1965, p. 7o. Zum gleichen Ergebnis in bezug auf Steinringe in Gletschervorfeldern gelangte auch $\mathrm{H}$. Stingl in einer bisher unveröffentlichten Seminararbeit am Geographischen Institut der Universität Göttingen: In 15 Gletschervorfeldern der Ostalpen fand er vornehmlich Steinringe mit Steinkern anstelle einer mehr oder weniger aussortierten Feinerdesäule, die echte Steinringe oder -polygone unbedingt aufweisen müssen.). - Am gleichen Institut entstand die bisher nicht veröffentlichte Habilitationsschrift von P. Höllermann. In dieser wird der (Periglazialformenschatz〉 der Pyrenäen und der Ostalpen behandelt. 


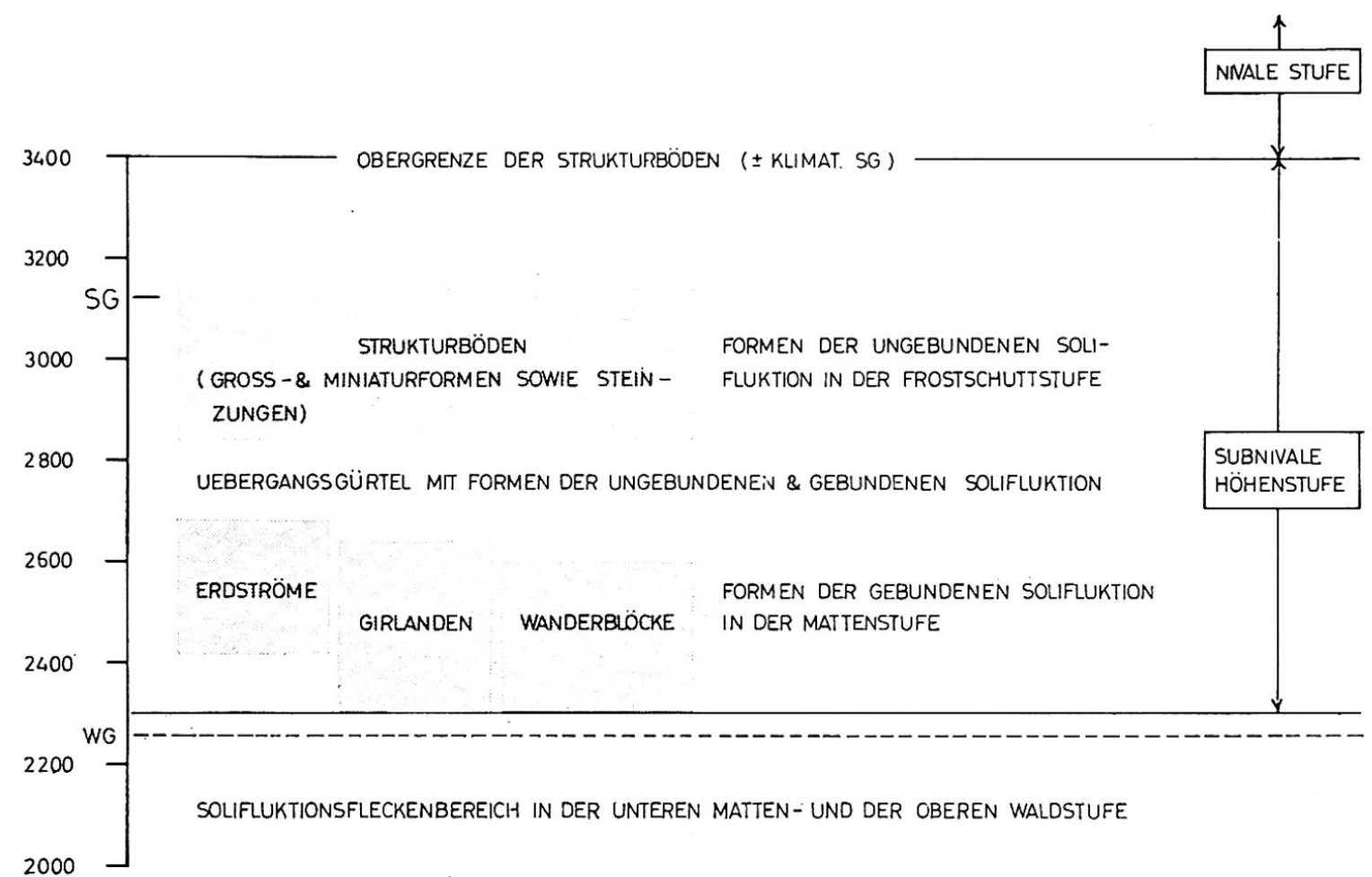

Fig. 1. Höhenlage von subnivalen Bodenformen, Raum Zermatt, Nordexposition (vgl. Furrer 1965, Abb. 44, 45 und 46). WG: Waldgrenze, gestützt auf Schroeter 1926. SG: klimatische Schneegrenze nach Jegerlehner 1902. Punktierte Flächen: Kernzonen verschiedener Formtypen. (In ihrer Kernzone treten die Formen am häufigsten auf; dort liegen auch die Hauptmasse der am klarsten ausgebildeten Formen und die größten zusammenhängend gemusterten Flächen.)

\section{Die Strukturböden der Frostschuttstufe}

Auf praktisch horizontalen Flächen treten Steinringe und -polygone auf (Abb. 1), die in der Regel Netze bilden. Geneigte Flächen hingegen tragen Steinovale, -zungen und-streifen; sie sind also mit den Strukturbodenformen der ungebundenen (〈freien〉) Solifluktion gemustert. Den Dimensionen nach lassen sich Groß-, Klein- und Miniaturformen von Strukturböden auseinanderhalten (Furrer 1965).

Steinnetze mit über 0,5 m Maschenweite (Großformen) werden nach den Beobachtungen von Büdel in Spitzbergen nur auf Flächen gebildet, deren Neigung weniger als $1,7-2^{\circ}$ beträgt (Büdel 1960, p. $29 ; 1961$, p. 358). Großformen von Steinstreifen lassen sich in den Alpen auf Hängen von $3-22^{\circ}$ Neigung erkennen. Gelegentlich noch bei $30-32^{\circ}$ Neigung auftretende Formen erwecken schon eher den Eindruck von (Spülrinnen〉. Es scheint, da $\beta$ an ihrer Bildung das fließende Wasser wesentlichen Anteil hatte. In bezug auf die Einregelung der Gesteinskomponenten (Stellung der Längsachsen zur Fallinie) ergeben sich zwar auch bei ausgefallener Steilheit dieselben charakteristischen Resultate wie bei den bevorzugten schwächeren Neigungen.

Bemerkenswert ist die Übereinstimmung der Einregelungsmessungen in den Steinstreifen. Über 50\% der Einzelsteine weichen normalerweise mit ihren Längsachsen weniger als $30^{\circ}$ von der Fallinie ab. Ein zweites, allerdings kleineres Maximum ergibt sich für die Abweichung der Längsachsen um $60-90^{\circ}$ von der Fallinie (vgl. Furrer 1965, p. 19). Neuere Beobachtungen scheinen eine ähnliche Regelmäßigkeit für Steinringe darzulegen, indem dort mehr als $50 \%$ der Einzelkomponenten bezüglich des Zentrums tangential angeordnet liegen und ein kleineres Maximum sich in radialer Richtung ausprägt. Eine Beziehung zwischen Grad der Hangneigung und Orientierung der Gesteinslängsachsen in Strukturbodenformen ließ sich bisher noch nicht ermitteln.

Die Erdstreifen, ein Miniaturtyp der Strukturböden, lassen sich noch auf Hangneigungen bis $35^{\circ}$ beobachten.

Strukturböden treten in der Frostschuttstufe nicht in allen Höhenlagen gleich häufig auf. Zwischen 2850 und $3130 \mathrm{~m}$ ü. M. ließ sich im Raume Zermatt die größte Häufigkeit beobachten. Dieser Höhenbereich kann somit als Kernzone ihrer Verbreitung auf- 


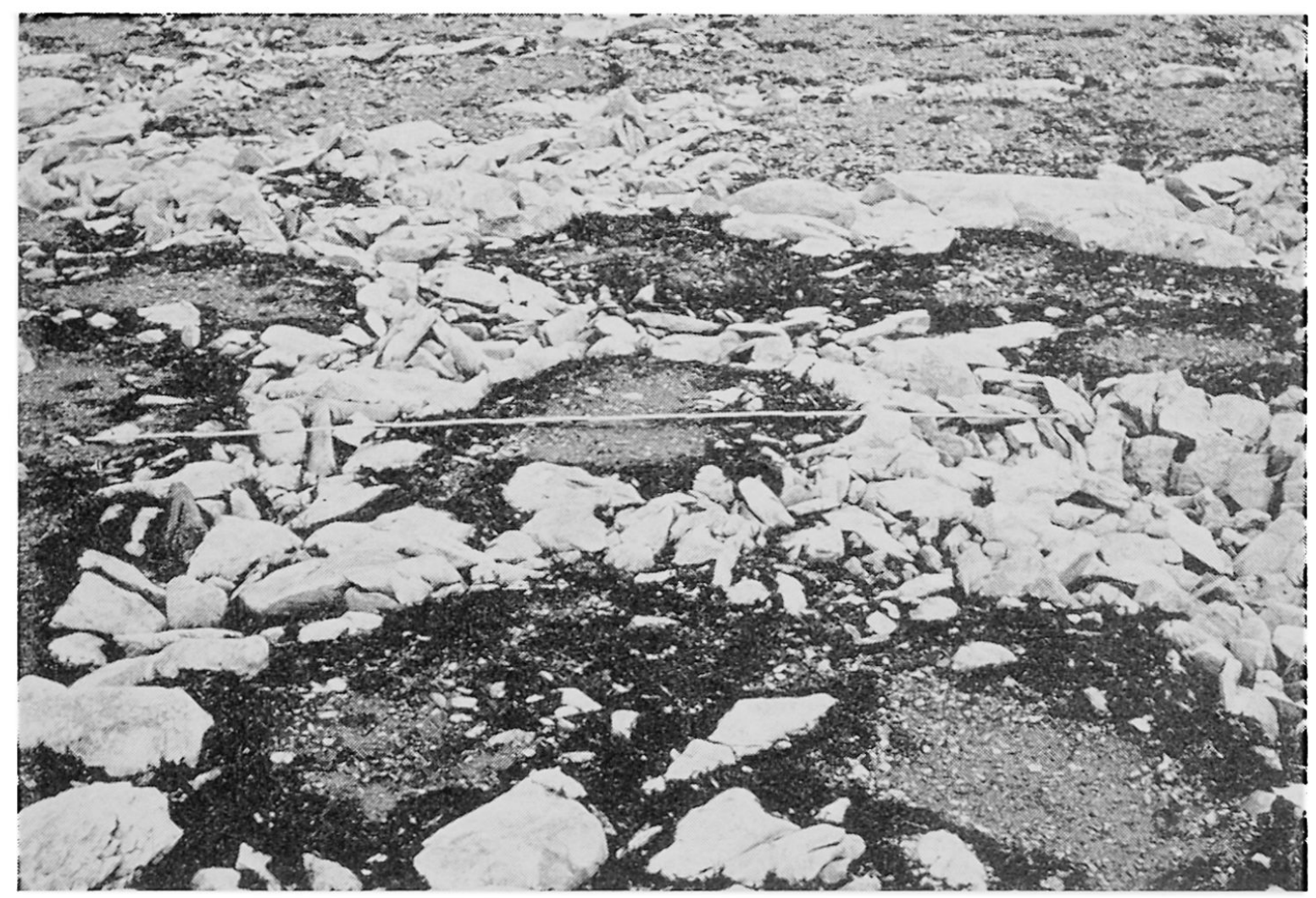

Abb. 1. Steinnetz mit deutlich ausgebildetem Steinring (Bildmitte). Cassonsgrat ob Flims (Landeskarte Blatt 247: 739100/193800, 266o m. Maßstab $2 \mathrm{~m}$ ). Kalktrümmer, die Vegetation setzt am Rand der Feinerdesäule an. Beachte die Orientierung der Steinlängsachsen im Steinring: vorwiegend tangential und daneben einige in radialer Lage.

gefaßt werden. Höher und tiefer liegende Vorkommen werden den Grenzsäumen zugeordnet. Nach den bisherigen Beobachtungen scheint die vertikale Höhenausdehnung der Kernzone sowohl im Wallis als auch im Bündnerland knappe $300 \mathrm{~m} z u$ betragen, also relativ konstant zu bleiben (Fig. 1 und 2).

Der untere Grenzsaum weist auffallend wenig Strukturbodenvorkommen auf, der obere hingegen wesentlich mehr, und vor allem zeichnet er sich durch zahlreiche prächtig ausgebildete Großformen aus. Die Obergrenze des gesamten Verbreitungsgebietes läßt sich in der Umgebung des Matterhorns auf Grund mehrerer Vorkommen eindeutig auf $3400 \mathrm{~m}$ ü. M. festlegen (Furrer 1965, Abb. 44).

\section{Strukturböden und Schneegrenze}

Auffallend erscheint, daß die Obergrenze der Kernzone, besonders aber die Obergrenze des gesamten Verbreitungsgebietes der Strukturböden, über der um die Jahrhundertwende von Jegerlehner (1902) festgelegten «klimatischen〉 Schneegrenze liegt (vgl. Fig. 1).

Jegerlehners Arbeit - nach der Methode von Kurowski durchgeführt — basiert auf der mittleren Höhe der Gletscheroberflächen, wie sie auf den der Arbeit zugrunde liegenden Kartenblättern aus der zweiten Hälfte des letzten Jahrhunderts verzeichnet waren. Seither sind die Gletscheroberflächen wesentlich zusammengeschrumpft. Abgesehen davon, daß Zweifel am Aussagewert der nach Kurowskis Methode gewonnenen Werten bestehen (Zingg 1954, p. 849), hat sich die Schneegrenze inzwischen beträchtlich nach oben verschoben (vgl. auch Streiff-Becker 1947 , p. 110).

Die in den vergangenen zehn Jahren gewonnenen Beobachtungen deuten darauf hin, daß Strukturböden heute in unseren Alpen in der hoch- bis spätsommerlichen Schnee- 


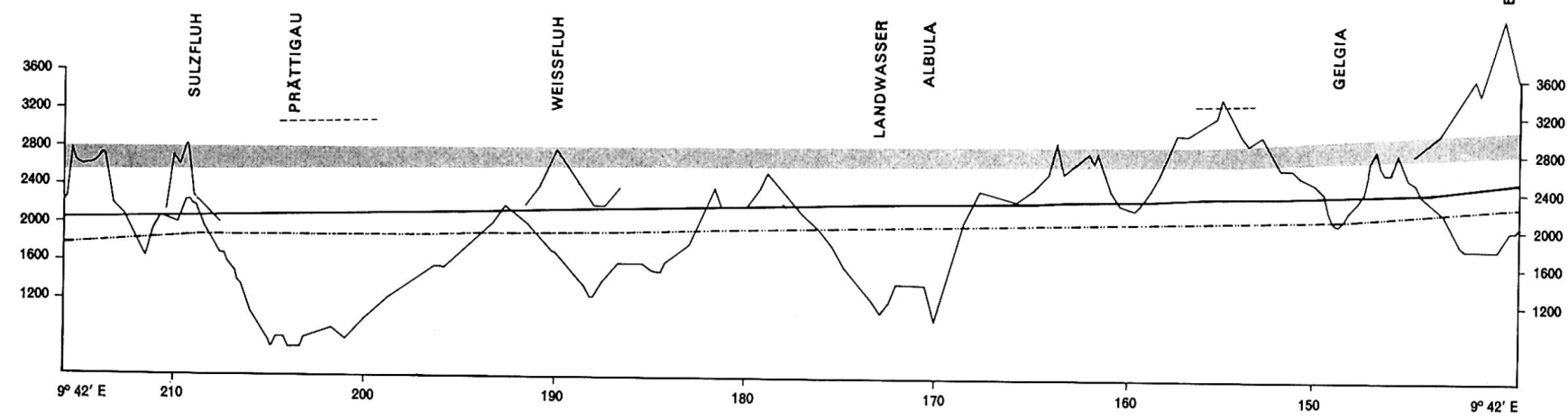

Figur 2 Verlauf von Höhengrenzen auf $9^{0} 42^{\prime} \mathrm{E}$ durch Graubünden

Strichpunktierte Linie: Waldgrenze nach Schroeter 1926. Kräftig ausgezogene Linie: Untergrenze der subnivalen Höhenstufe.
Punktierter Streifen: Strukturbodenkernzone (Groß- und Miniaturformen sowie Steinzungen).

Gestrichelte Linien: klimatische Schneegrenze. 


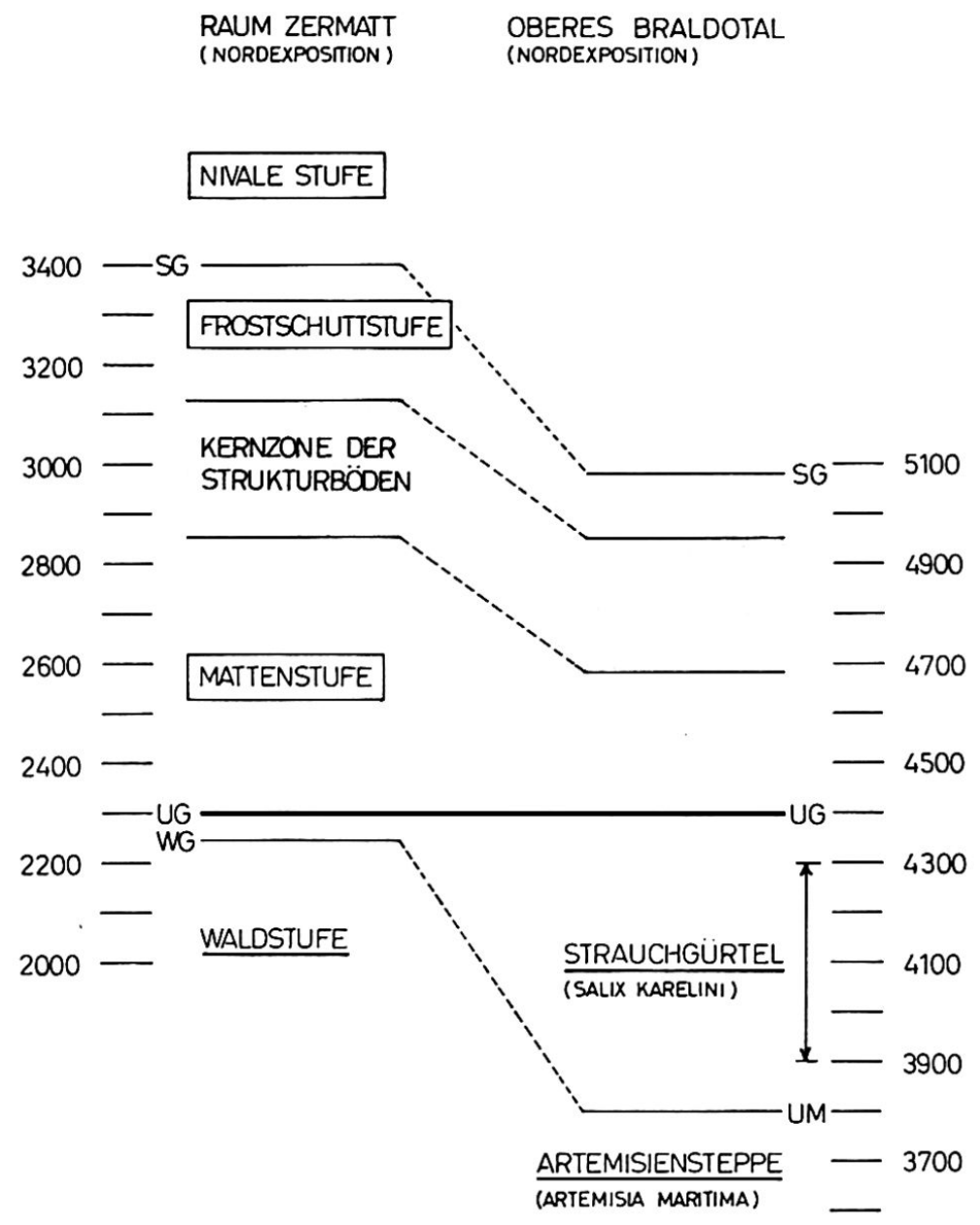

Figur 3

Vergleich zwischen den subnivalen Höhenstufen im Raum Zermatt und im oberen Braldo- und Biafotal (Karakorum).

UM: untere Grenze der Mattenstufe.

WG: Waldgrenze nach Schroeter 1926.

UG: Untergrenze der subnivalen Höhenstufe.

SG: klimatische Schneegrenze.

fleckenregion gebildet werden. Zu Beginn der Schneeschmelze sind beispielsweise die Steine in den Steinstreifen (Großformen) noch fest zusammengefroren. Die Frostbodenoberfläche verläuft in dieser Periode gleich wie in den Strukturbodenformen Spitzbergens (vgl. Furrer 1959, Fig. 7 und 8). Mit fortschreitender Schneeschmelze bildet sich die Auftauzone. Die Frostbodenoberfläche wird sukzessive tiefer gelegt, und über dem noch vorhandenen Frostboden, der sich allenfalls jahrelang erhalten kann, entsteht Fließerde. Solange während dieses Auftauprozesses die aufgetaute Masse noch stark durchfeuchtet ist, können in den Flächen zwischen den Steinstreifen unregelmäßig erfolgende Fließvorgänge beobachtet werden. Im Spätsommer und im Herbst ist je nach Höhenlage und Feuchtigkeitsverhältnissen in den Strukturböden der Frost aktiv.

Wenn Strukturböden höhenwärts spärlicher auftreten, hängt dies weitgehend mit der Verarmung der Böden an Feinerde und mit der Reliefungunst - Steilheit der Hänge und Mangel an horizontalen Flächen - zusammen. Es stellt sich die Frage, ob die Strukturbodenformen dank ihrer relativ kurzen Bildungsdauer einer höherrückenden Schneegrenze in kurzem zeitlichem Abstand zu folgen vermögen. Dann vermöchten besonders die höchstgelegenen Steinnetze auf horizontalen Flächen einen Anhaltspunkt über die Höhenlage der klimatischen Schneegrenze zu geben. Diese liegt im Raume von Zermatt, nach der oberen Strukturbodengrenze beurteilt, mindestens um 300 Meter höher, als sie 1902 durch Jegerlehner festgelegt worden ist (Fig. 1).

\section{Der Höhenbereich der Formen der gebundenen Solifluktion}

Während die Strukturböden ihre eindrücklichste Ausprägung und ihre größte Verbreitung in der Frostschuttstufe erlangen, finden sich die Kernzonen der gebundenen Solifluktionsformen in der Mattenstufe. Diesem Bereich zugehörige Formen lassen sich 
aber bereits im untern Grenzsaum der Strukturbodenstufe erkennen, der zusammen mit dem obern Grenzsaum des Hauptverbreitungsgebietes der Formen der gebundenen Solifluktion einen Übergangsgürtel zwischen den subnivalen Bodenformen der Frostschutt- und der Mattenstufe bildet. Es handelt sich um die Höhenlage, in der die vorwiegend geschlossene Pflanzendecke der Mattenstufe sich in Vegetationsinseln aufzulösen beginnt.

Die Untergrenze des Hauptverbreitungsgebietes der Formen der gebundenen Solifluktion konnte am Alpennordrand bei gut $2000 \mathrm{~m}$ festgelegt werden; sie steigt aber gegen die Hauptwasserscheide im Bündnerland auf über $2400 \mathrm{~m}$ hinauf (Fig. 2).

Unterhalb der Kernzone gebundener Solifluktionsformen lassen sich Girlanden und Wanderblöcke oft bis weit in die Waldstufe hinunter antreffen. Wir ordnen diese Vorkommen dem Solifluktionsfleckenbereich zu.

\section{Die Untergrenze der subnivalen Höhenstufe, festgelegt mit Hilfe des subnivalen For- menschatzes}

Die subnivale Höhenstufe reicht meiner Auffassung nach so weit talwärts, als die subnivalen Formen wesentlich an der Kleinreliefgestaltung der Hochgebirgslandschaft beteiligt sind (vgl. dazu auch Troll 1955 und Poser 1957). Die Grenzziehung anhand der Formen wird um so zuverläßiger, je mehr Beobachtungen vorliegen. Sie stützt sich auf zahlreiche Kartierungen, wobei extrazonale Vorkommen und Pseudoformen ausgesondert werden müssen.

Im gewählten Beispiel (Fig. 1) fallen die Untergrenzen der Kernzonen von Girlanden und Wanderblöcken zusammen. So ist die Festlegung der gesuchten Grenze einfach, indem diese beiden Linien die Hauptverbreitungsgebiete aller Solifluktionsformen abschließen. Viel häufiger decken sich aber diese beiden Kernzonenuntergrenzen nicht, oder dann lassen sich die eine, schlimmstenfalls sogar beide mangels Formen für ein bestimmtes Gebiet überhaupt nicht bestimmen.

Von den drei Hauptvertretern der gebundenen Solifluktionsformen liegen nach allen bisherigen Beobachtungen die Kernzonen der Erdströme höher als jene der Girlanden und Wanderblöcke (Furrer 1965, p. 58). Zur talwärtigen Abgrenzung der subnivalen Höhenstufe bieten sich daher die beiden letzteren an. So wurde mit Hilfe ihres Hauptverbreitungsgebietes die in Figur 2 dargestellte Grenze ermittelt. Da nicht geklärt ist, ob die in einigen Regionen Graubündens hektarenweit verbreiteten Erdbülten - zum Beispiel auf dem Dreibündenstein - Ruheformen darstellen, wurden diese zur Grenzziehung nicht herangezogen.

\section{Die vertikale Ausdehnung der subnivalen Höhenstufe}

Aus den Figuren 1 und 2 erhellt die $Z$ weiteilung des alpinen Solifluktionsbereiches. Unsicher ist der Verlauf der klimatischen Schneegrenze. Ihre minimale Höhenlage wurde auf Grund beobachteter Solifluktionsvorgänge und Strukturbodenformen eingezeichnet. Sie dürfte wohl eher zu tief angesetzt sein (mündliche Mitteilung von Herrn Dr. Zingg, Eidg. Institut für Schnee- und Lawinenforschung).

Mit Hilfe von Figur 2 ermittelt, besitzt die subnivale Höhenstufe in den Bündner Alpen eine mittlere Vertikalerstreckung von 1000 m. Im kleineren Raume um Zermatt, auf der Nordseite der Hauptwasserscheide, wo die dieser Arbeit zugrunde liegenden Kartierungen sich dichter scharen als in Bünden und sehr viel Beobachtungsmaterial vorliegt, erhöht sich der Wert auf $1100 \mathrm{~m}$.

Dieser steigt nach Bär und Leemann im westlichen Hohen Atlas mindestens auf $1600 \mathrm{~m}$ (1963 und mdil. Mitt.).

Für das östliche Mittelmeergebiet gibt Poser (1957) den Wert von 1200-13oo m für den Abstand zwischen der Schneegrenze und der Untergrenze des (periglazialen) Bereiches an. Im oberen Braldo- und Biafotal (Karakorum) läßt siç die Untergrenze der subnivalen Höhenstufe, 
gestützt auf deutlich entwickelte Formen, bei Htoom ü. M. beobachten (Furrer 1965, Abb. 49). Bei Annahme der Schneegrenze zwischen 5000 und $5100 \mathrm{~m}$ resultiert ein entsprechender Wert von nur $600-700 \mathrm{~m}$. Dieser ist $z w a r$ mit verschiedenen Unsicherheiten behaftet und daher nicht gleich repräsentativ wie die erwähnten Werte aus unseren Alpen.

Doch geben die Beobachtungen im Bradotal Aufschluß über die Verhältnisse in einer Trokkenregion. Allerdings können sie, da sonst noch keine derartigen Beispiele untersucht worden sind, kaum Anspruch auf Allgemeingültigkeit erheben. Um $2400 \mathrm{~m}$ ü. M. bedecken im asiatischen Arbeitsgebiet weite Sandfluren und Dünen den Talboden. Nach dem Vegetationsbefund müssen mit wachsender Höhe die Niederschläge zunehmen. Messungen liegen keine vor. Der subnivale Formenschatz ist in der gleichen Höhenabfolge angeordnet wie in unseren Alpen (Furrer 1965, p. 6o). In beiden Arbeitsgebieten ist die Höhenerstreckung der Strukturbodenkernzone praktisch dieselbe (Zermatt $280 \mathrm{~m}$, oberes Braldotal $250 \mathrm{~m}$; Fig. 3). Hingegen schrumpft die Stufe der Formen der gebundenen Solifluktion gegenüber den alpinen Verhältnissen zusammen.

Im Braldotal zieht die Untergrenze an den nordexponierten Hängen rund $100 \mathrm{~m}$ über dem Strauchgürtel durch. Ihre relativ hohe Lage steht somit nicht in Beziehung mit der erwähnten Vegetationsstufe (indem die tief wurzelnden Sträucher die Solifluktion hemmen würden), sondern muß dadurch erklärt werden, daß die tieferen Lagen der zur Ausbildung von Solifluktionsformen erforderlichen Feuchtigkeit entbehren. Dies wird durch die Beobachtungen an südexponierten Hängen - denen der Strauchgürtel allgemein fehlt — bestätigt: Im oberen Braldotal liegt die Untergrenze des subnivalen Bereiches auf südexponierten Hängen $50 \mathrm{~m}$ höher als auf nordexponierten, am Matterhorn gute $100 \mathrm{~m}$. Es ergibt sich also in beiden Gebieten auch eine weitgehende Übereinstimmung in bezug auf die Abhängigkeit von der Exposition.

Der Solifluktionsfleckenbereich greift im Braldotal in die obersten Lagen der Artemisiensteppe hinunter. Dort lassen sich vereinzelte, allerdings nicht optimal ausgebildete Girlanden erkennen, an deren Stirnen jedoch nicht die vertrauten Pflanzenarten der Alpen zu finden sind.

\section{Zusammenfassung}

1. In der vorliegenden Arbeit wird die Untergrenze des Hauptverbreitungsgebietes der Solifluktionsformen als Untergrenze der subnivalen Höhenstufe aufgefaßt. Diese liegt in der Mattenstufe und verläuft im gleichen Sinne wie die Waldgrenze, doch nicht streng parallel dazu. Generalisierend kann auf Grund der gegenwärtig vorliegenden Beobachtungen vom Rätikon bis zur Berninagruppe einerseits, vom Alpennordrand am Thunersee (Niesen) bis zur Südseite des Matterhorns anderseits - also über eine Strecke von annähernd $80 \mathrm{~km}$ - ein Ansteigen dieser Untergrenze von 2000 auf über $2400 \mathrm{~m}$ festgestellt werden.

2. Figur 1 gibt eine mögliche Gliederung der subnivalen Stufe wieder.

3. Die bisherigen Ergebnisse lassen erkennen, daß die von Jegerlehner erarbeitete und in verschiedenen Abhandlungen zur Geographie der Alpen aufgeführte Höhenlage der klimatischen Schneegrenze den heutigen Verhältnissen kaum mehr gerecht wird. Neue Höhenangaben von Klimatologen über den Verlauf dieser Grenze wären für klimamorphologische Arbeiten sehr wertvoll.

4. Das «Bildungsmilieu〉 der subnivalen Bodenformen - insbesondere das Material des Bodens und dessen Vegetationsbedeckung sowie Relief-, Expositions- und Feuchtigkeitsverhältnisse - kann auf kleinstem Raume wechseln. Es gestattet in der subnivalen Höhenstufe die Ausbildung der verschiedenen Formtypen nicht überall in gleicher Weise. Bis andere brauchbare Methoden vorliegen, sind darum möglichst viele Kartierungen und Feldbeobachtungen nötig, um trotzdem die Verbreitungsgebiete des subnivalen Formenschatzes abgrenzen zu können.

\section{IN DIESER ARBEIT BENÜTZTE LITERATUR}

Büdel, J. (1960) : Die Frostschutt-Zone Südost-Spitzbergens. Colloquium Geographicum 6; Büdel, J. (1961): Die Abtragungs-Vorgänge auf Spitzbergen im Umkreis der Barents-Insel. Deutscher Geographentag Köln 1961 (Sonderdruck), S. 337-375. - Furrer, G. (1959) : Untersuchungen am subnivalen Formenschatz in Spitzbergen und in den Bündner Alpen. Geographica Helvetica 4, S. 277-309; - Furrer, G. (1965): Die Höhenlage von subnivalen Bodenformen. Arbeiten aus dem Geographischen Institut der Universität Zürich (Habilitationsschrift). Höllermann, P. (1964): Rezente Verwitterung, Abtrag und Formenbildung im oberen Suldental 
(Ortlergruppe/Südtirol). Diss. Göttingen (Zeitschrift für Geomorphologie, N. F. Supplementband 4). - Jegerlehner, J. (19o2): Die Schneegrenze in den Gletschergebieten der Schweiz. Gerland's Beiträge zur Geophysik V/3, S. 486-566. - Poser, H. (1954) : Die Periglazial-Erscheinungen in der Umgebung der Gletscher des Zemmgrundes (Zillertaler Alpen). Göttinger Geographische Abhandlungen 15, S. 125-180) ; - Poser, H. (1957) : Klimamorphologische Probleme auf Kreta. Zeitschrift für Geomorphologie 2, S. 113-142. - Schroeter, C. (1926): Das Pflanzenleben der Alpen. - Streiff-Becker, R. (1947) : Die Schneegrenzen in den Alpen. Leben und Umwelt 7, S. 107-11o. - Troll, C. (1944): Strukturböden, Solifluktion und Frostklimate der Erde. Geologische Rundschau (Klimaheft), S. 545-694; - Troll, C. (1947) : Die Formen der Solifluktion und die periglaziale Bodenabtragung. Erdkunde I, S. 162-175; - Troll, C. (1948) : Der subnivale oder periglaziale Zyklus der Denudation. Erdkunde II, S. 1-21; - Troll, C. (1955): Über das Wesen der Hochgebirgsnatur. Jahrbuch des Österreichischen Alpenvereins. - Zingg, T. (1954): Die Bestimmung der klimatischen Schneegrenze auf klimatologischer Grundlage. Mitteilungen des Eidg. Institutes für Schnee- und Lawinenforschung 12. - Bär und Leemann (1963): Klimamorphologische Untersuchungen in Marokko. Vierteljahresschrift der Naturforschenden Gesellschaft in Zürich. Heft 3, 295-357.

\section{THE SUBNIVEAL ZONE AND ITS LOWER DELIMINATION IN THE ALPS OF THE GRISONS AND THE VALAIS}

This essay takes the lower boundary of the occurrence of solifluction phenomena as the lower limit of the subniveal zone. This lies within the Alpine pasture belt and though not necessarily parallel, is analagous to the timberline. Observations from the northern perimeter of the Alps (Lake of Thun) to the Matterhorn and from Rätikon to the Bernina (a distance of some 5o miles), show this lower bound to ascend from 2000 metres to over 2400 metres. However, the exact delimination of subniveal forms needs further field-observation and mapping.

\section{PORTO}

\section{WERNER NIGG}

Der Name Portos ist dank des vorzüglichen und einzigartigen Weines, des Vinho do Porto, in aller Welt bekannt. Doch wissen verhältnismäßig wenige Ausländer Näheres über die Stadt, die diesem edlen Tropfen den Namen gegeben hat. Das läßt sich zum Teil damit erklären, daß Lissabon als Kapitale vor allen andern portugiesischen Städten eine unbestreitbare Vorrangstellung einnimmt und wegen seiner Lage und seinem Klima eine stärkere Anziehungskraft ausübt als Porto. Zwischen diesen beiden einzigen Großstädten Portugals bestanden seit jeher Rivalitäten, und Porto wurde nicht selten von Lissabon als unbequeme Nebenbuhlerin empfunden.

Porto liegt am rechten Ufer des Rio Douro, der sich hier ein tiefes, schluchtartiges Tal eingefressen hat und $5 \mathrm{~km}$ weiter westwärts in den Atlantischen Ozean mündet. Die Stadt dehnt sich über einen 60-70 $\mathrm{m}$ hohen Granitrücken aus und steigt mit ihren alten, enggebauten Quartieren über den steilen Hang zum Douro hinunter.

Auf dem gegenüberliegenden Ufer liegt Vila Nova de Gaia, ein großer Vorort, in dem sich die meisten Lager der berühmten Portweinfirmen befinden. Zwei imposante Brücken, ehrwürdige Eisenkonstruktionen, überspannen ca. $60 \mathrm{~m}$ über dem Wasserspiegel mit eleganten Bögen das Tal: die 1876-1877 von der bekannten Pariser Firma Eiffel erstellte, $352 \mathrm{~m}$ lange Ponte de Maria Pia mit einem Bogen von $160 \mathrm{~m}$ Spannweite und die 1881-1885 von der belgischen Société des Constructions de Willebroek erbaute Ponta de Dom Luiz I. mit 170 m Bogenspannweite. Diese beiden Brücken wirken besonders im Gegenlicht wie großartige Filigranmuster und gehören zu den Wahrzeichen Portos. Vor einigen Jahren ist zudem für die nach Süden führende Autobahn eine weitere leistungsfähige Brücke dem Betrieb übergeben worden. 